\title{
A COMPREHENSIVE RIEMANNIAN FRAMEWORK FOR THE ANALYSIS OF WHITE MATTER FIBER TRACTS
}

\author{
Meena Mani ${ }^{1}$, Sebastian Kurtek ${ }^{2}$, Anuj Srivastava ${ }^{2}$, Christian Barillot ${ }^{1}$ \\ ${ }^{1}$ Visages Project, IRISA/INRIA Rennes, France \\ ${ }^{2}$ Department of Statistics, Florida State University, Tallahassee, Florida, USA
}

\begin{abstract}
A quantitative analysis of white matter fibers is based on different physical features (shape, scale, orientation and position) of the fibers, depending on the specific application. Due to the different properties of these features, one usually designs different metrics and spaces to treat them individually. We propose a comprehensive Riemannian framework that allows a joint analysis of these features in a consistent manner. For each combination, we provide a formula for the distance, i.e. quantification of differences between fibers and a formula for geodesics, i.e. optimal deformations of fibers into each other. We illustrate this framework in the context of clustering fiber tracts from the corpus callosum and study the results from different combinations of features.
\end{abstract}

\section{INTRODUCTION}

The analysis of white matter fibers, of interest in applications as varied as the differential diagnosis of white matter diseases, neurosurgery or a study of brain connectvity, requires different techniques and approaches. Some of the more recent Diffusion Tensor MRI (DT-MRI) literature has addressed issues such as the clustering of fibers into anatomically meaningful bundles [1], atlas building for group studies [2] and statistical methods for quantitative analysis [3] or to evaluate fractional anisotropy, mean diffusivity and other measures of white matter integrity along tracts [4].

Geometrically, white matter fibers reconstructed using DTMRI, can be described as 3-dimensional open, continuous curves. These fibers, when viewed as curves, have certain physical features associated with them, namely shape, scale, orientation, and position. There have been several recent papers on shape analysis of continuous curves using a Riemannian framework [5]. This type of framework has many advantages: (1) It provides techniques for comparing, matching, and deforming shapes of curves under the chosen metric. The correspondences for these tasks are established automatically. (2) It also provides tools for defining and computing statistical summaries of sample shapes for different shape

Meena Mani was supported in part by an Université Européenne de Bretagne (UEB) grant. classes [6]. In the current problem, there is interest in including the other features - scale, orientation, and position - in the analysis, since this information can significantly aid the clustering, classifying, labeling and quantitative analysis of fiber tracts. It is therefore desirable to have a comprehensive Riemannian framework that can compare and quantify differences between these multiple features in a coherent way. In this conceptual paper, we define a sequence of feature spaces, each associated with a Riemannian metric, such that any desired combination of features of fibers can be analyzed using geodesics and geodesic distances. An important aspect of this work is that despite dealing with parameterized curves, the resulting geodesics and geodesic distances are invariant to the actual parametrization of the curves.

The rest of this paper is organized as follows. The mathematical framework for comparing curves using different features is laid out in Section 2. Experimental results demonstrating feasibility are presented in Section 3, and some concluding remarks are presented in Section 4.

\section{MATHEMATICAL FRAMEWORK}

Let $\beta:[0,2 \pi] \rightarrow \mathbb{R}^{3}$ be a parameterized curve, representing a fiber tract. We are interested in developing a Riemannian framework where we can study different physical features associated with $\beta$. An important requirement in this analysis is to obtain results that do not depend on any particular parameterization of $\beta$. If we use the standard $\mathbb{L}^{2}$ metric for comparing curves $\left(\left\|\beta_{1}-\beta_{2}\right\|_{2}=\sqrt{\int_{0}^{2 \pi}\left\|\beta_{1}(t)-\beta_{2}(t)\right\|^{2} d t}\right)$, as is often done in quantitative analysis of fibers, then the results do not have the independence to the re-parameterizations. This forces us to choose novel mathematical representations of curves that allows us to have the desired invariance. In the following, we are going to present a sequence of representations (spaces and metrics) that capture different combinations of features and provide techniques for comparing curves according to those features. In particular, we are going to provide two things in each case: (i) a geodesic distance between curves that depends only on selected features (and is independent of the parameterization of curves), and (ii) a geodesic path between the two curves. The geodesic paths are useful for many 
reasons. Firstly, it provides a way of deforming one curve into the other in an optimal way. Secondly, it is fundamental to computing sample statistics, such as means and covariances, of curves.

\subsection{Comparison using the complete feature set}

We start by considering a situation where we are interested in comparing curves using all the four physical features - shape, scale, position and orientation. Define a representation of a curve $\beta$ using a square-root function (SRF):

$$
h(t)=\sqrt{\|\dot{\beta}(t)\| \beta}(t), \quad h:[0,2 \pi] \rightarrow \mathbb{R}^{3} .
$$

In order to compare any two curves, we will compare their SRFs. The metric that we use for this comparison is the $\mathbb{L}^{2}$ metric. One advantage of this representation is its invariance to re-parameterization of curves. We explain this point further. Let $\gamma:[0,2 \pi] \rightarrow[0,2 \pi]$ be a smooth, one-to-one, and onto function; $\gamma$ serves as a re-parameterization function for any curve. (Let $\Gamma$ denote the set of all such functions.) For a curve $\beta$, the new curve $\tilde{\beta}(t) \equiv \beta(\gamma(t))$ is simply the old curve with a new parameterization. Also, for the re-parameterized curve $\tilde{\beta}$, the SRF is given by $\tilde{h}(t)=\sqrt{\dot{\gamma}(t)} h(\gamma(t))$. We often use $(h, \gamma)$ to denote this re-parameterized SRF. Now, it can be shown that for any two curves $\beta_{1}, \beta_{2}$, with the corresponding SRFs $h_{1}$ and $h_{2}$, and any $\gamma \in \Gamma$, we have that $\left\|\left(h_{1}, \gamma\right)-\left(h_{2}, \gamma\right)\right\|_{2}=\left\|h_{1}-h_{2}\right\|_{2}$. Because of this equality, we can define a distance between the two curves as:

$$
d_{a}\left(\beta_{1}, \beta_{2}\right)=\min _{\gamma \in \Gamma}\left(\left\|h_{1}-\left(h_{2}, \gamma\right)\right\|_{2}\right)
$$

This minimization is performed using the standard dynamic programming (DP) algorithm, and it results in a quantification of differences in curves that is associated with all four features - shape, position, orientation, and scale. In addition to a proper distance, this framework also provides a geodesic path between the curves. In this case, the geodesic path is given by:

$$
\psi(\tau)=(1-\tau) h_{1}+\tau\left(h_{2}, \gamma^{*}\right),
$$

where $\gamma^{*}$ is the optimal re-parameterization obtain earlier in minimization using DP.

\subsection{Comparison using shape, scale and orientation}

Suppose we want to compare curves using all features except their positions. Now we consider the task of comparing curves using three features (shape, orientation, and scale) and thus not including the position in the analysis. In this case we define a new representation of $\beta$, using the velocity function $\dot{\beta}$, as follows: Given $\beta:[0,1] \rightarrow \mathbb{R}^{3}$, define

$$
q(t)=\frac{\dot{\beta}(t)}{\sqrt{\|\dot{\beta}(t)\|}}, q:[0,2 \pi] \rightarrow \mathbb{R}^{3} .
$$

This function is different from the earlier square-root function in that this definition is completely based on the velocity function $\dot{\beta}$. Therefore, this function is called the square-root velocity function (SRVF) [7]. Since this function is invariant to a global translation of $\beta$, any analysis based on this function will not dependent on the global coordinates of the curves. Note that the norm $\|q(t)\|$ defines the square root of the instantaneous speed along the curve $\beta$. It is important to know that one can recover the original curve $\beta$, within a translation, using $\beta(t)=\int_{0}^{t}\|q(s)\| q(s) d s$. If $\tilde{\beta}=\beta \circ \gamma$ is a re-parameterized curve, then its $\operatorname{SRVF}$ is given by $(q, \gamma) \equiv$ $\sqrt{\dot{\gamma}(t)} q(\gamma(t))$, where $q$ is the SRVF of the original curve.

As earlier, it can be shown that for any curves $\beta_{1}, \beta_{2}$, with the corresponding SRVFs $q_{1}$ and $q_{2}$, and any $\gamma \in \Gamma$, we have that $\left\|\left(q_{1}, \gamma\right)-\left(q_{2}, \gamma\right)\right\|_{2}=\left\|q_{1}-q_{2}\right\|_{2}$. Once again, we can define a distance between the two curves as:

$$
d_{b}\left(\beta_{1}, \beta_{2}\right)=\min _{\gamma \in \Gamma}\left\|q_{1}-\left(q_{2}, \gamma\right)\right\|_{2} .
$$

This minimization is performed using the DP algorithm, and it results in a quantification in differences in curves according to the remaining three features - shape, orientation, and scale. In this case, the geodesic path between the two curves is given by:

$$
\psi(\tau)=(1-\tau) q_{1}+\tau\left(q_{2}, \gamma^{*}\right),
$$

where $\gamma^{*}$ is the optimal re-parameterization obtain the earlier minimization.

\subsection{Comparison using shape and orientation}

Now we consider a situation where the scales of observed curves are not important and we want to remove them from the analysis. In order to remove the influence of scales of curves in the quantitative analysis, we can rescale them to be of the same length, say 1 . The mathematical representation and the Riemannian metric remains same as earlier except the set of SRVFs is reduced as a consequence of this rescaling. If the curve $\beta$ is of length 1 , then $\int_{0}^{1}\|\dot{\beta}(t)\| d t=$ $\int_{0}^{1}\|q(t)\|^{2} d t=1$ holds. Therefore, the set of all SRVF functions associated with curves of length one are elements of a hypersphere in $\mathbb{L}^{2}$ (since their norms are one). This greatly simplifies the shape analysis due to the fact that the differential geometry of a sphere is well-known. For example, if $q_{1}$ and $q_{2}$ are two elements of a unit hypersphere, the geodesic distance between them is given by length of shortest arc connecting them on the sphere. This length is actually given by $\cos ^{-1}\left(\int_{0}^{2 \pi}\left\langle q_{1}(t), q_{2}(t)\right\rangle d t\right)$. As in the previous two cases, this distance does not depend on the re-parameterization of the two curves. That is, for any $q_{1}, q_{2}$ and $\gamma$,

$$
\begin{gathered}
\cos ^{-1}\left(\int_{0}^{2 \pi}\left\langle q_{1}(t), q_{2}(t)\right\rangle d t\right)= \\
\cos ^{-1}\left(\int_{0}^{2 \pi}\left\langle\left(q_{1}, \gamma\right)(t),\left(q_{2}, \gamma\right)(t)\right\rangle d t\right) .
\end{gathered}
$$


This leads to the definition of a distance between two curves that depends only on their shapes and orientations:

$d_{c}\left(\beta_{1}, \beta_{2}\right)=\min _{\gamma \in \Gamma}\left(\cos ^{-1}\left(\int_{0}^{2 \pi}\left\langle\left(q_{1}, \gamma\right)(t),\left(q_{2}, \gamma\right)(t)\right\rangle d t\right)\right)$.

In this case, the geodesic path between the two curves is given by:

$$
\psi(\tau)=\frac{1}{\sin (\theta)}\left[\sin (\theta-\tau \theta) q_{1}+\sin (\tau \theta)\left(q_{2}, \gamma^{*}\right)\right],
$$

where $\theta=d_{c}\left(\beta_{1}, \beta_{2}\right)$.

\subsection{Comparison using shape and scale}

As the next case, we are interested in comparing curves according to their shapes and scales, i.e. we want to remove the rigid motions from the representations. Let $S O(3)$ the set of all possible rotations in $\mathbb{R}^{3}$. If we rotate the curve $\beta$ by a rotation matrix $O \in S O(3)$, we obtain the curve $O \beta(t)$. The SRVF of the rotated curve is given by $O q$ where $q$ is the SRVF of the original curve. Consequently, the SRVF of a rotated and re-parameterized curve is given by $\sqrt{\dot{\gamma}(t)} O q(\gamma(t))$. To include the scale, we return to the SRVF representation of unscaled curves, and the distance function given in Eqn. 2 applies. The SRVFs are no longer restricted to a sphere but are elements of the full space. In order to remove the orientation feature, we need to add the minimization over $S O(3)$ as follows. Define a distance function:

$$
d_{d}\left(\beta_{1}, \beta_{2}\right)=\min _{\gamma \in \Gamma, O \in S O(3)}\left\|q_{1}-O\left(q_{2}, \gamma\right)\right\|_{2} .
$$

Let $\gamma^{*}$ and $O^{*}$ be the re-parameterization and the rotation that minimize the right side in this equation. Then, the geodesic path between any two curves is given by:

$$
\psi(\tau)=(1-\tau) q_{1}+\tau\left(O^{*} q_{2}, \gamma^{*}\right) .
$$

\subsection{Comparison using shape only}

In the final case, we are interested in comparing curves only according to their shapes. That is, we want to remove all other physical variables (positions, scales, and orientations) from the representations, and want to consider only the influence of shapes in clustering and classification of fibers. The geodesic distance between any two scaled SRVFs is given by Eqn. 3. In order to remove orientation, we have to minimize over $S O(3)$ as well as $\Gamma$ now. Define a distance function:

$d_{e}\left(\beta_{1}, \beta_{2}\right)=\min _{\gamma \in \Gamma, O \in S O(3)} \cos ^{-1}\left(\int_{0}^{2 \pi}\left\langle\left(q_{1}, \gamma\right)(t), O\left(q_{2}, \gamma\right)(t)\right\rangle d t\right)$.

Let $\gamma^{*}$ and $O^{*}$ be the re-parameterization and the rotation that minimize the right side in this equation. Then, the geodesic path between any two curves is given by: for $\theta=d_{e}\left(\beta_{1}, \beta_{2}\right)$,

$$
\psi(\tau)=\frac{1}{\sin (\theta)}\left[\sin (\theta-\tau \theta) q_{1}+\sin (\tau \theta)\left(O^{*} q_{2}, \gamma^{*}\right)\right] .
$$

\section{CLUSTERING CORPUS CALLOSAL FIBERS}

As an application, we consider the clustering of fibers of the corpus callosum using distances derived from the methods described above.

The corpus callosum(CC) is a large collection of fibers that connect the left and right hemispheres (Fig. 1). Pathologies such as multiple sclerosis [8], schizophrenia and Alzheimer's disease [9] selectively affect specific regions of the $\mathrm{CC}$ and so there is interest in segregating the different regions for study. Clustering is made difficult both because there are no obvious landmarks and also because a limitation in DT-MRI tractography [10] renders fibers which have small differences in shape and scale along the length of the CC.

Combining shape, orientation and scale feature distances gives better discrimination than shape information alone. In the case of the $\mathrm{CC}$, the genu(blue) and splenium(green) are at opposite ends and are easily grouped using the joint shape, orientation and scale metric, $d_{b}$ (Fig.2a). The results for shape clustering alone (using $d_{e}$ ) are shown in Fig. 2b for comparison.

There are instances where a combination of shape, orientation and scale do not yield the best results. When a third bundle, the anterior section of the corpus(red) is added for clustering, the joint shape and orientation distance, $d_{c}$, consistently gives better results than the $d_{b}$ metric (Fig. 3). This can be explained by the fact that the genu(blue) and corpus(red) have roughly the same scale and tend to cluster together with a $d_{b}$ metric. They are, however, oriented in different directions $\left(0^{\circ}, 90^{\circ}, 180^{\circ}\right.$ respectively) and so the $d_{c}$ metric, which does not include scale, provides better discrimination.

A second example is the clustering of the isthmus(magenta) and the splenium(green) at the posterior end of the CC(Fig. 4). The scale parameter, similar for the two groups, dominates the clustering and using either the shape and scale, $d_{d}$, or the $d_{b}$ distance gives poor results. The $d_{c}$ metric, which does not include scale information, gives the best results (Fig. 4a).

\section{CONCLUSION}

This conceptual paper presents a comprehensive Riemannian framework for comparing fiber tracts using a variety of physical variables - shapes, positions, orientations, and scales. Depending on the application, some combination of these variables may be sufficient for a quantitative analysis. We consider a number of these combinations and for each combination we provide: (i) a geodesic distance function for comparing curves according to the chosen features, (ii) a geodesic path equation for computing geodesic paths between given curves. Both these quantities are invariant to the re-parameterizations of curves. This framework is versatile, in that, it allows for inclusion of any combination of shape, scale, orientation and translation in the analysis. The primary application of this framework analysis of 3D open curves is classification of DT- 
MRI white matter fibers in the brain. This framework can be extended to include additional features, e.g. the fractional anisotropy function and the mean diffusivity function along the fibers, to further strengthen the quantitative analysis [11].

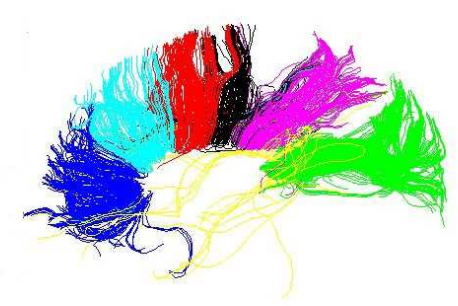

Fig. 1. A mid-sagittal view of the corpus callosum. The rostrum and genu(blue), rostral body(cyan), anterior corpus(red), posterior corpus(black), isthmus(magenta), tapetum(yellow) and splenium(green) subdivisions are based on the Witelson [12] classification.

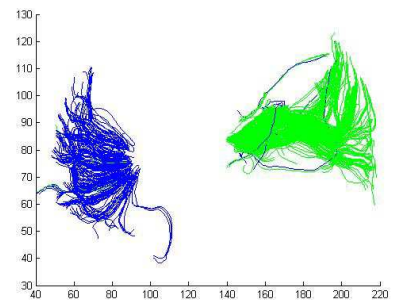

(a) shape+orientation+scale $\left(d_{b}\right)$

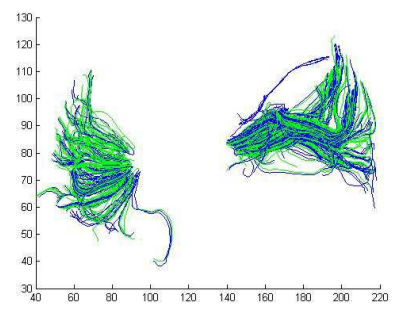

(b) shape $\left(d_{e}\right)$
Fig. 2. Clustering the genu and splenium, the anterior and posterior sections of the CC. Here, shape information alone (2b) is not adequate for clustering.

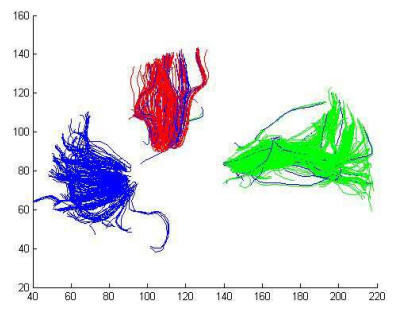

(a) shape+orientation $\left(d_{c}\right)$

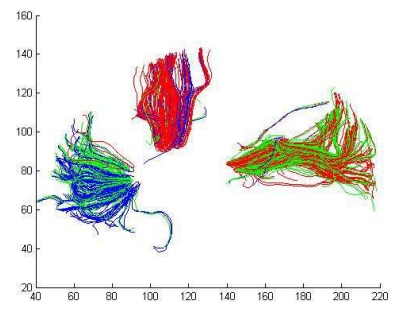

(b) shape+orientation+scale $\left(d_{b}\right)$
Fig. 3. Clustering of the genu, corpus and splenium, the anterior, middle and posterior sections of the CC. Including the scale information results in poorer clustering (3b).

\section{REFERENCES}

[1] Song Zhang et. al, "Identifying white-matter fiber bundles in dti data using an automated proximity-based fiber-clustering method," IEEE Trans. on Visualization and Computer Graphics, vol. 14, no. 5, pp. 1044-1053, 2008.

[2] Lauren J. O'Donnell et. al, "Automatic Tractography Segmentation Using a High-Dimensional White Matter Atlas," IEEE Transactions on Medical Imaging, vol. 26, no. 11, pp. 15621575, November 2007.

[3] M. Maddah et. al, "A Unified Framework for Clustering and Quantitative Analysis of White Matter Fiber Tracts," Med Image Anal, 042008.

[4] Casey B. Goodlett et. al, "Group analysis of dti fiber tract statistics with application to neurodevelopment," NeuroImage, vol. 45, no. 1, Supplement 1, pp. S133 - S142, 2009.

[5] L. Younes et. al, "A metric on shape space with explicit geodesics," Matematica E Applicazioni, vol. 19, no. 1, pp. 25-57, 2008.

[6] A. Srivastava et. al, "Statistical shape analysis: Clustering, learning and testing," IEEE Trans. on Pattern Analysis and Machine Intelligence, vol. 27, no. 4, pp. 590-602, 2005.

[7] S. H. Joshi et. al, "A novel representation for Riemannian analysis of elastic curves in $\mathrm{R}^{\mathrm{n}}$," in CVPR, 2007.

[8] N. Evangelou et. al, "Regional axonal loss in the corpus callosum correlates with cerebral white matter lesion volume and distribution in multiple sclerosis," Brain, vol. 123, no. 9, pp. 1845-1849, 2000.

[9] Denise Head et. al, "Differential Vulnerability of Anterior White Matter in Nondemented Aging with Minimal Acceleration in Dementia of the Alzheimer Type: Evidence from Diffusion Tensor Imaging," Cereb. Cortex, vol. 14, no. 4, pp. 410423, 2004.

[10] Michio Ono et. al, Atlas of the Cerebral Sulci, Thieme Medical Publisher, 1990.

[11] W. Liu et. al, "Joint shape and texture analysis of objects boundaries in images using a Riemannian approach," in Asilomar Conferences on Signals, Systems, and Computers, 2008.

[12] Sandra F. Witelson, "Hand and Sex Differences in the Isthmus and Genu of the Human Corpus Callosum: A Postmortem Morphological Study," Brain, vol. 112, no. 3, pp. 799-835, 1989.

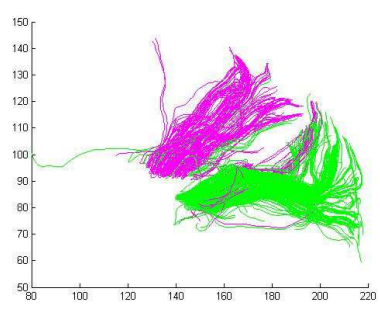

(a) shape+orientation $\left(d_{c}\right)$

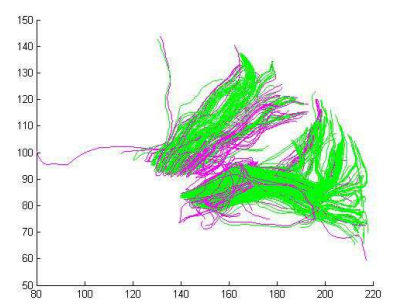

(b) shape+scale $\left(d_{d}\right)$
Fig. 4. Clustering the isthmus and splenium, the posterior CC. 